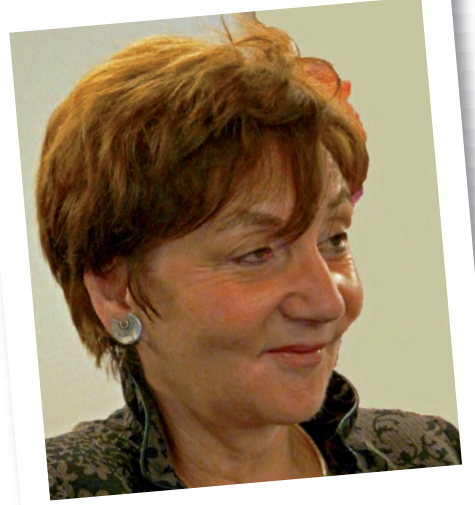

\title{
Des temps difficiles
}

En ces périodes de contestation dans le milieu universitaire, la SFP n’a pas échappé aux turbulences. Son président Yves Petroff, nouvellement nommé, a démissionné le 3 mars à la suite de désaccords internes dans le contexte de discussions sur un éventuel rachat d'EDP Sciences par Springer. Cette affaire nous a valu de très nombreux messages ; l'enchaînement des événements vous est rapporté dans l'article pp. 29-30. Les négociations autour de cette vente sont maintenant arrêtées, avant même d’avoir vraiment commencé, et je souhaite vivement que la SFP retrouve rapidement toute sa sérénité.

Je tiens à dire d’abord que je déplore vivement, et tout le bureau de la SFP avec moi, le départ d'Yves Petroff. II avait pris avec beaucoup de vigueur des initiatives très positives pour la SFP, comme en témoigne son éditorial d'investiture dans le précédent numéro de Reflets. La SFP a beaucoup bénéficié de sa vision à long terme, alimentée par sa grande expérience de la recherche au niveau international. Qu'il soit ici remercié du travail qu'il a accompli pour notre société pendant toute son année de vice-présidence. En attendant qu’une personnalité se dégage parmi nous pour reprendre le flambeau, jai accepté d’assurer de nouveau la présidence de la SFP pendant quelque temps.

Sans tomber dans le pessimisme qui domine en cette période de crise, il faut admettre que la situation est tendue dans le milieu universitaire. Les projets ministériels se succèdent à un rythme épuisant : dans le secondaire, puis sur les carrières dans le supérieur, et maintenant pour la "mastérisation " de la formation des enseignants. Sur toutes ces questions la SFP a travaillé, en concertation étroite avec les sociétés de mathématiques (SMF) et de chimie (SCF), pour développer des analyses, critiquer les projets, faire des propositions d'amendement, intervenir par des lettres ou des entrevues avec les ministres concernés. Toutes les positions de la SFP sont consultables sur son site (www.sfpnet.fr, page « débats »). Dans certains cas, comme pour les projets sur la classe de seconde ou la réécriture du décret sur l'évaluation des enseignantschercheurs, nous espérons avoir un peu contribué au recul du gouvernement sur les points les plus contestables des réformes envisagées.

Pour ce qui est de la SFP elle-même, je partage les préoccupations exposées par Yves Petroff dans son éditorial. Le nombre des membres de notre société plafonne à un chiffre ridiculement bas ; la tendance est même plutôt à la baisse, comme l'indique le graphique fourni plus loin (p. 28) par notre trésorier. L'adhésion à prix très réduit pour les jeunes a certes attiré quelques centaines de doctorants, mais une grande proportion des chefs d'équipe et des directeurs de laboratoire ne se sent toujours pas motivée pour adhérer à notre société, en dépit de nos actions généralisées pour promouvoir la science. De plus, notre pénétration dans le milieu des entreprises reste très faible. Renforcer la SFP est un grand chantier qui prendra du temps, car il s'agit d'établir une tradition d'adhésion en motivant les jeunes dès le début de leur carrière, comme à l'IOP ou à la DPG, les sociétés de physique britannique et allemande. On peut aussi espérer que la création d'une fédération plus large, regroupant des sociétés d'ingénieurs avec la SFP, apportera une partie de la solution.

Élargir la base de ses membres est le moyen le plus sûr pour la SFP d'accroître et même de conserver sa visibilité. En effet, il faut être conscient que la source de revenus de la SFP provenant d'EDP Sciences est susceptible de diminuer dans l'avenir : les ventes des revues scientifiques chez EDP sont à la baisse, les bibliothèques américaines résilient leurs abonnements avec la crise, les revues en open access font concurrence. Sans revenir sur l'occasion manquée du rachat d'EDP par Springer, il est clair qu'il va falloir trouver de nouvelles idées pour soutenir notre filiale d'édition et maintenir son potentiel, ce qui est aussi important pour son personnel que pour la SFP elle-même.

Michèle Leduc

Présidente par intérim de la Société Française de Physique 